\title{
咖啡因、茶碱和氨茶碱的低温热容和热分析
}

\author{
徐芬 ${ }^{1,2}$ 邱树君 ${ }^{4}$ 梁建国 ${ }^{3}$ 吴瑞华 ${ }^{4}$ 孙立贤 ${ }^{2}$ * 李 芬 ${ }^{2}$ \\ ( ${ }^{1}$ 辽宁师范大学化学化工学院, 辽宁大连 116029; ${ }^{2}$ 中国科学院大连化学物理研究所, 辽宁 大连 116023; \\ 3 湖南省药品检验所, 长沙 $410001 ; 4$ 辽宁省大连市药品检验所, 辽宁大连 116021)
}

\begin{abstract}
摘要：咖啡因、茶碱和氨茶碱是临床应用中广泛使用的三种重要的甲基化的黄嘌呤. 采用绝热量热、热重分析 (TG)和差示扫描量热法(DSC)研究了这三种药物的热力学性质. 采用绝热量热法测定了 $\beta$ 型-咖啡因、茶碱和氨 茶碱在 80-370 K 温度范围内的摩尔热容值, 结果显示氨茶碱的摩尔热容值最大, 茶碱的摩尔热容值最小. 采用 最小二乘法对这三种药物热容的测量值和温度进行了拟合, 得到了热容与折合温度的多项式, 计算了咖啡因、茶 碱和氨茶碱在 $298.15 \mathrm{~K}$ 时的热容分别为 $226.49 、 218.13$ 和 $554.78 \mathrm{~J} \cdot \mathrm{K}^{-1} \cdot \mathrm{mol}^{-1}$; 并计算了它们相对于 $298.15 \mathrm{~K}$ 时 的焓和熵. 采用热综合分析仪对这三种药物的热稳定性进行了评价, 结果表明它们的热稳定顺序为氨茶碱<咖啡 因<茶碱. 通过 DSC 分析, 得到了咖啡因和茶碱的相转变温度、相转变焓和熵. 基于密度泛函理论的第一性原理, 计算了咖啡因和茶碱分子的结构稳定性, 结果显示咖啡因分子的稳定性低于茶碱, 与实验结果吻合.
\end{abstract}

关键词：低温热容; 咖啡因; 茶碱; 氨茶碱; 热分析

中图分类号: 0642.3

\section{Low Temperature Heat Capacity and Thermal Analysis of Caffeine, Theophylline and Aminophylline}

\author{
XU Fen ${ }^{1,2} \quad$ QIU Shu-Jun ${ }^{4} \quad$ LIANG Jian-Guo ${ }^{3} \quad$ WU Rui-Hua ${ }^{4} \quad$ SUN Li-Xian ${ }^{2, *} \quad$ LI Fen $^{2}$ \\ $\left({ }^{1}\right.$ College of Chemistry and Chemical Engineering, Liaoning Normal University, Dalian 116029, Liaoning Province, P. R. China; \\ ${ }^{2}$ Dalian Institute of Chemical Physics, Chinese Academy of Sciences, Dalian 116023, Liaoning Province, P. R. China; \\ ${ }^{3}$ Hunan Institute of Drug Detection, Changsha 410001, P. R. China; ${ }^{4}$ Dalian Institute of Drug Detection, Dalian 116021,
} Liaoning Province, P. R. China)

\begin{abstract}
Caffeine, theophylline, and aminophylline are important methyl-substituted xanthines and are widely used in clinics. In this work, the thermodynamic characteristics of the three drugs were studied by adiabatic calorimetry, thermogravimetric analysis (TG) and differential scanning calorimetry (DSC). The low temperature molar heat capacities of caffeine (in the $\beta$ crystal form), theophylline and aminophylline were measured by heating the system from 80 to $370 \mathrm{~K}$ using an adiabatic calorimeter. The results indicate that the molar heat capacity of aminophylline is the largest while that of theophylline is the smallest. The experimental molar heat capacities of the three drugs were fitted to a polynomial of $C_{p, \mathrm{~m}} v s$ the reduced temperature $(t)$ by means of the least fitting square method from 80 to 370 $\mathrm{K}$. Their molar heat capacities at $298.15 \mathrm{~K}$ were calculated to be $226.49 \mathrm{~J} \cdot \mathrm{K}^{-1} \cdot \mathrm{mol}^{-1}$ (for caffeine), $218.13 \mathrm{~J} \cdot \mathrm{K}^{-1} \cdot \mathrm{mol}^{-1}$ (for theophylline), and $554.78 \mathrm{~J} \cdot \mathrm{K}^{-1} \cdot \mathrm{mol}^{-1}$ (for aminophylline) using the polynomial $C_{p, \mathrm{~m}}-t$. Thermodynamic parameters (such as enthalpies and entropies relative to $298.15 \mathrm{~K}$ ) were calculated for these drugs based on the polynomial $C_{p, \mathrm{~m}}-t$. The results of thermal analysis show that the order of thermal stability for these drugs is aminophylline $<$ caffeine $<$ theophylline. The temperatures, enthalpies and entropies of the phase transitions for caffeine and theophylline were
\end{abstract}

Received: March 26, 2010; Revised: April 16, 2010; Published on Web: June 30, 2010.

"Corresponding author. Email: 1xsun@ dicp.ac.cn; Tel/Fax: +86-411-84379213.

The project was supported by the National Natural Science Foundation of China (U0734005, 20833009, 20873148, 20903095), National Key Basic Research Program of China (973) (2010CB631303) and Liaoning Normal University Foundation, China.

国家自然科学基金(U0734005, 20833009, 20873148, 20903095), 国家重点基础研究发展规划项目(973) (2010CB631303)和辽宁师范大学基金 资助

(C) Editorial office of Acta Physico-Chimica Sinica 
obtained by DSC. The stabilities of the molecular structures for caffeine and theophylline were calculated by a firstprinciples calculation based on density functional theory. The results imply that the stability of the caffeine molecule is lower than that of theophylline and this is in good agreement with the experimental results.

Key Words: Low temperature heat capacity; Caffeine; Theophylline; Aminophylline; Thermal analysis

嘌呤在自然界中广泛存在, 并在人和动物的新 陈代谢中起着重要的作用. 咖啡因、茶碱和氨茶碱是 最重要的三种甲基化的黄嘌呤, 它们在临床上的应 用十分广泛. 人们对它们的应用研究产生了浓厚的 兴趣 ${ }^{[1-5]}$, 同时对这三种药物的含量测定方法也进行 了大量的研究 ${ }^{[-9-9]}$.

热分析和量热学已广泛应用于药学领域中 ${ }^{[10-13]}$. 如 Kunadharaju 等 ${ }^{[10]}$ 采用热分析方法研究了具有抗 癌作用的 10-差基喜树碱的热力学性质; 张健等 ${ }^{[11]}$ 采 用热分析法研究了喹诺酮类药物的热稳定性. 对咖 啡因和茶碱的一些热力学性质也有一些报道. 文 献报道 ${ }^{[14-16]}$ 了咖啡因晶体存在两种晶型 $(\alpha$ 型和 $\beta$ 型), 并测量了其固-固相变、固-液相变的温度和焓 变值, 但不同文献报道的数据间存在一些差异, 如 Cesàro 等 ${ }^{[14]}$ 测量的固-固相变温度为 $426 \mathrm{~K}$, 而 Bruns 等 ${ }^{[16]}$ 报道的为 $141{ }^{\circ} \mathrm{C}(414.15 \mathrm{~K})$, 因此, 有必要 对咖啡因的相变过程再进行测量. Dong 等 ${ }^{[17]}$ 测量了 $\alpha$-咖啡因的热容和燃烧热, 并计算了其生成焓和相 关的热力学参数. Yoshihashi 等 ${ }^{[183}$ 测量了茶碱的脱

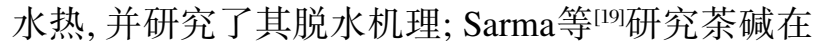
等温 $\left(40\right.$ 和 $\left.47^{\circ} \mathrm{C}\right)$ 条件下的热力学性质, 在等温差热 分析 (DTA) 曲线中观察到茶碱 $\left(\mathrm{C}_{7} \mathrm{H}_{8} \mathrm{~N}_{4} \mathrm{O}_{2} \cdot \mathrm{H}_{2} \mathrm{O}\right)$ 脱水的吸热峰. 但目前尚未见茶碱和氨茶碱热容值 及有关比较这三种药物热力学性质的报道.

众所周知, 物质的热容、熵、焓等是非常重要的 热力学参数. 而物质的熵、焓等均可由其热容值的大 小推导出来. 为此, 有许多科学工作者在研究物质热 容的测量方法, 目前文献报道较多的是采用绝热量 热方法测量各种物质的热容值 ${ }^{[20-25]}$.

本文采用绝热量热仪、热综合分析仪, 系统地研 究咖啡因、茶碱和氨茶碱的热力学性质, 通过绝热量 热的方法获得这些药物的热容值及相关的热力学参 数, 丰富药品的热力学数据; 而其热分析结果对于 药品的生产与保存具有一定的指导价值.

\section{1 实验和理论计算方法}

\section{1 试剂与仪器}

咖啡因(石家庄制药集团)、茶碱和氨茶碱(山东 新华制药股份有限公司)均由湖南省药品检验所提 供, 其纯度符合中国药典标准(均大于 99.0\%). 其中 热容测定结果表明本文所研究的咖啡因在固-固相 变 $(426 \mathrm{~K})$ 前为 $\beta$-型.

绝热量热计由中国科学院大连化学物理研究所 材料热化学研究组自行研制.

\section{2 样品低温热容值的测定}

低温热容测定在自制的小样品精密自动绝热量 热装置中进行, 该法的测量原理及仪器结构参见文 献 ${ }^{[23]}$. 该测量的温度精度为 $0.001 \mathrm{~K}$, 其范围为 $80-$ $370 \mathrm{~K}$, 加热速率控制在 $0.2-0.4 \mathrm{~K} \cdot \mathrm{min}^{-1}$ 之间, 液氮 作为冷却剂. 在量热实验中, 所有测试数据均由计算 机自动采集和适时处理. 为了证实该绝热热量计测 量结果的准确性, 预先测量了量热参考标准物质 $\alpha$ $\mathrm{Al}_{2} \mathrm{O}_{3}$ 在 $80-370 \mathrm{~K}$ 温度区间的摩尔热容, 实验数据 和美国标准及技术研究院(NIST)提供的数据 ${ }^{[26]}$ 之间 的相对偏差在 $\pm 0.80 \%$ 以内.

\section{3 样品的热分析}

采用法国 Setaram 公司生产的 Setsys 16/18 热 综合分析仪对样品进行热分析. Setsys 16/18 综合分 析仪的实验条件为: 控制升温速率为 $10 \mathrm{~K} \cdot \mathrm{min}^{-1} ; \mathrm{N}_{2}$ 气作为载气, 其流速控制为 $30 \mathrm{~mL} \cdot \mathrm{min}^{-1}$; 温度范围 为室温至 $750 \mathrm{~K}$. 通过该实验可获得样品的热失重 曲线( TG 曲线). 该仪器的温度及焓值用金属标准样 品(铟、锡、铅和铝)进行标定, 其温度测量的相对误 差小于 $0.8 \%$, 焓值测量的相对误差小于 $3 \%$; 并用标 准草酸钙 $\left(\mathrm{Ca}\left(\mathrm{C}_{2} \mathrm{O}_{4}\right)_{2} \cdot \mathrm{H}_{2} \mathrm{O}\right)$ 对仪器的热失重精度进行 校正, 其相对误差小于 $1.5 \%$.

\section{4 理论计算方法}

基于密度泛函理论 ${ }^{[27]}$ 的第一性原理, 对咖啡因 和茶碱分子的结构稳定性进行了计算, 采用全电子 计算的 $\mathrm{DMol}^{3}$ 程序 ${ }^{[28]}$. 选用了广义梯度近似下的 PW91 泛函 ${ }^{[2]}$ 来处理交换关联作用,采用 TNP 数值 基组以及 $50 \mathrm{~nm}$ 的截断半径, 对所有结构都进行了 完全驰豫的结构优化. 
<smiles>CC1C(=O)c2c(ncn2C)N(C)C1=O</smiles><smiles></smiles><smiles>CC1C(=O)c2c(ncn2[InH])N(C)C1=O</smiles>

图 1 三种甲基黄嘌呤的分子结构

Fig.1 Molecular structures of three kinds of methyl-substituted xanthines

\section{2 结果与讨论}

\section{1 咖啡因、茶碱和氨茶碱的低温热容的测定}

图 1 为咖啡因、茶碱和氨茶碱的分子结构. 从图 1 中可看出, 咖啡因和茶碱的区别仅在于 7 号位上 $\mathrm{N}$ 原子相连的取代基, 一个与- $\mathrm{CH}_{3}$ 相连, 另一个与 $\mathrm{H}$ 相连. 而氨茶碱为茶碱与乙二胺的复合盐.

分别称取咖啡因 $2.8016 \mathrm{~g}$ 、茶碱 $1.7861 \mathrm{~g}$ 和氨茶 碱 $1.0594 \mathrm{~g}$, 按文献描述的方法 ${ }^{[23]}$ 进行热容测量, 实 验结果见表 1-3 及图 2 .

从图 2 可看出: 咖啡因、茶碱和氨茶碱在测量 的温度范围内均为一光滑曲线. 这表明在此温度范 围没有相变和任何其它热异常现象发生, 且茶碱与
乙二胺形成的盐一一氨茶碱的摩尔热容值最大, 而 茶碱的摩尔热容值最小. 由于甲基的引人, 使得咖啡 因的摩尔热容值略大于茶碱.

本文采用最小二乘法, 在 80-370 K 温度范围内 分别对上述三种药物的实验摩尔热容值与折合温度 $t(t=T / 1000, T$ 单位为 $\mathrm{K})$ 进行拟合, 得到它们的摩尔 热容与折合温度的关系式 (其中 $R^{2}$ 值均大于 0.9995). 对于咖啡因, 其摩尔热容与折合温度的关 系式为:

$$
\begin{aligned}
C_{p, \mathrm{~m}}= & -13.89337+1699.508 t-4988.859 t^{2}+ \\
& 6684.143 t^{3}
\end{aligned}
$$

而对于茶碱, 其摩尔热容与折合温度的关系式为:

表 1 咖啡因的摩尔热容测量值

\begin{tabular}{|c|c|c|c|c|c|c|c|c|c|}
\hline \multirow{2}{*}{$T / \mathrm{K}$} & $C_{p, \mathrm{~m}}$ & \multirow{2}{*}{$T / \mathrm{K}$} & $C_{p, \mathrm{~m}}$ & \multirow{2}{*}{$T / \mathrm{K}$} & \multirow{2}{*}{$\frac{C_{p, \mathrm{~m}}}{\left(\mathrm{~J} \cdot \mathrm{mol}^{-1} \cdot \mathrm{K}^{-1}\right)}$} & \multirow{2}{*}{$T / \mathrm{K}$} & \multirow{2}{*}{$\frac{C_{p, \mathrm{~m}}}{\left(\mathrm{~J} \cdot \mathrm{mol}^{-1} \cdot \mathrm{K}^{-1}\right)}$} & \multirow{2}{*}{$T / \mathrm{K}$} & \multirow{2}{*}{$\frac{C_{p, \mathrm{~m}}}{\left(\mathrm{~J} \cdot \mathrm{mol}^{-1} \cdot \mathrm{K}^{-1}\right)}$} \\
\hline & $\overline{\left(\mathrm{J} \cdot \mathrm{mol}^{-1} \cdot \mathrm{K}^{-1}\right)}$ & & $\overline{\left(\mathrm{J} \cdot \mathrm{mol}^{-1} \cdot \mathrm{K}^{-1}\right)}$ & & & & & & \\
\hline 77.535 & 91.365 & 138.219 & 142.85 & 185.523 & 172.78 & 250.087 & 204.80 & 326.038 & 240.83 \\
\hline 79.906 & 94.041 & 140.423 & 144.68 & 187.445 & 173.87 & 253.606 & 205.40 & 329.021 & 242.90 \\
\hline 84.419 & 98.646 & 142.614 & 145.45 & 189.390 & 174.57 & 257.124 & 206.46 & 332.004 & 246.01 \\
\hline 88.167 & 102.19 & 144.785 & 146.90 & 191.312 & 175.04 & 260.642 & 208.48 & 334.987 & 246.74 \\
\hline 91.073 & 104.44 & 146.941 & 149.23 & 193.225 & 176.23 & 264.084 & 210.84 & 338.046 & 248.50 \\
\hline 93.973 & 107.24 & 149.080 & 150.60 & 195.134 & 177.38 & 267.526 & 211.56 & 341.029 & 250.36 \\
\hline 96.771 & 110.68 & 151.205 & 151.11 & 197.035 & 178.32 & 270.968 & 212.50 & 343.936 & 252.12 \\
\hline 99.507 & 112.87 & 153.315 & 153.35 & 198.930 & 179.25 & 274.334 & 214.75 & 346.842 & 254.82 \\
\hline 102.191 & 115.26 & 155.411 & 153.82 & 200.819 & 180.94 & 277.775 & 217.24 & 349.596 & 255.96 \\
\hline 104.825 & 117.09 & 157.495 & 155.98 & 202.704 & 181.78 & 281.064 & 217.48 & 352.349 & 258.14 \\
\hline 107.415 & 118.83 & 159.564 & 157.35 & 204.577 & 182.63 & 284.430 & 219.26 & 354.950 & 259.28 \\
\hline 109.961 & 121.05 & 161.621 & 158.30 & 206.414 & 183.26 & 287.718 & 221.03 & 357.397 & 261.14 \\
\hline 112.474 & 123.24 & 163.668 & 159.58 & 209.320 & 184.92 & 290.931 & 222.69 & 359.692 & 263.01 \\
\hline 114.950 & 125.97 & 165.702 & 161.01 & 213.298 & 186.46 & 294.067 & 224.58 & 361.834 & 264.15 \\
\hline 117.397 & 127.30 & 167.726 & 162.94 & 217.198 & 189.47 & 297.279 & 226.84 & 363.746 & 266.22 \\
\hline 119.807 & 129.38 & 169.741 & 163.51 & 221.099 & 191.42 & 300.492 & 227.46 & 365.352 & 267.57 \\
\hline 122.193 & 131.37 & 171.743 & 164.49 & 224.924 & 191.79 & 303.628 & 229.22 & 366.729 & 268.92 \\
\hline 124.551 & 133.60 & 173.736 & 165.89 & 228.627 & 194.10 & 306.840 & 229.84 & 367.723 & 269.74 \\
\hline 126.884 & 135.42 & 175.721 & 167.63 & 232.266 & 196.63 & 309.976 & 231.60 & 368.411 & 270.05 \\
\hline 129.193 & 136.00 & 177.698 & 168.02 & 235.861 & 197.53 & 313.112 & 233.88 & & \\
\hline 131.483 & 137.72 & 179.668 & 169.76 & 239.456 & 199.00 & 316.248 & 235.13 & & \\
\hline 133.748 & 139.99 & 181.627 & 170.30 & 242.974 & 200.54 & 319.384 & 237.16 & & \\
\hline 135.992 & 141.84 & 183.579 & 171.31 & 246.493 & 203.03 & 322.290 & 238.24 & & \\
\hline
\end{tabular}

Table 1 Experimental molar heat capacity of caffeine 
表 2 茶碱的热容测量值

Table 2 Experimental molar heat capacity of theophylline

\begin{tabular}{|c|c|c|c|c|c|c|c|c|c|}
\hline$T / \mathrm{K}$ & $\frac{C_{p, \mathrm{~m}}}{\left(\mathrm{~J} \cdot \mathrm{mol}^{-1} \cdot \mathrm{K}^{-1}\right)}$ & $T / \mathrm{K}$ & $\frac{C_{p, \mathrm{~m}}}{\left(\mathrm{~J} \cdot \mathrm{mol}^{-1} \cdot \mathrm{K}^{-1}\right)}$ & $T / \mathrm{K}$ & $\frac{C_{p, \mathrm{~m}}}{\left(\mathrm{~J} \cdot \mathrm{mol}^{-1} \cdot \mathrm{K}^{-1}\right)}$ & $T / \mathrm{K}$ & $\frac{C_{p, \mathrm{~m}}}{\left(\mathrm{~J} \cdot \mathrm{mol}^{-1} \cdot \mathrm{K}^{-1}\right)}$ & $T / \mathrm{K}$ & $\frac{C_{p, \mathrm{~m}}}{\left(\mathrm{~J} \cdot \mathrm{mol}^{-1} \cdot \mathrm{K}^{-1}\right)}$ \\
\hline 77.764 & 76.633 & 142.236 & 112.67 & 209.091 & 156.16 & 264.084 & 194.85 & 314.642 & 228.18 \\
\hline 79.371 & 77.387 & 144.763 & 114.05 & 211.232 & 158.09 & 265.920 & 195.85 & 316.401 & 229.76 \\
\hline 81.895 & 78.941 & 147.269 & 115.35 & 213.298 & 159.21 & 267.832 & 197.78 & 318.083 & 229.97 \\
\hline 84.358 & 79.732 & 149.750 & 117.72 & 215.349 & 161.10 & 269.668 & 198.11 & 319.766 & 231.43 \\
\hline 86.725 & 80.875 & 152.212 & 118.06 & 217.402 & 162.06 & 271.580 & 200.71 & 321.525 & 233.22 \\
\hline 89.038 & 82.188 & 154.655 & 120.97 & 219.450 & 163.23 & 273.416 & 202.12 & 323.208 & 234.09 \\
\hline 91.299 & 83.699 & 161.884 & 125.91 & 221.482 & 164.99 & 275.251 & 204.40 & 324.891 & 235.42 \\
\hline 93.521 & 85.016 & 164.256 & 127.51 & 223.547 & 166.10 & 277.087 & 204.91 & 326.650 & 235.42 \\
\hline 95.662 & 85.971 & 166.614 & 128.99 & 225.535 & 168.93 & 278.999 & 206.51 & 328.333 & 236.69 \\
\hline 97.804 & 86.274 & 168.952 & 130.13 & 227.524 & 169.26 & 280.835 & 207.00 & 330.015 & 237.89 \\
\hline 99.937 & 87.667 & 171.276 & 130.59 & 229.513 & 170.80 & 282.594 & 208.31 & 331.698 & 239.41 \\
\hline 102.010 & 89.205 & 173.592 & 132.69 & 231.501 & 172.62 & 284.430 & 209.24 & 333.381 & 241.20 \\
\hline 104.076 & 90.774 & 175.897 & 134.78 & 235.402 & 174.90 & 286.265 & 211.40 & 335.063 & 240.95 \\
\hline 106.064 & 91.034 & 178.184 & 135.19 & 237.391 & 176.06 & 288.101 & 212.87 & 336.746 & 241.69 \\
\hline 108.053 & 92.254 & 180.459 & 137.91 & 239.303 & 177.29 & 289.860 & 213.57 & 338.429 & 242.95 \\
\hline 109.965 & 93.039 & 182.723 & 138.28 & 241.215 & 179.26 & 291.619 & 213.96 & 340.035 & 243.82 \\
\hline 112.413 & 94.637 & 184.978 & 140.20 & 243.051 & 180.79 & 293.378 & 215.43 & 341.718 & 244.22 \\
\hline 115.319 & 96.303 & 187.222 & 141.27 & 244.963 & 181.25 & 295.138 & 216.30 & 343.324 & 245.88 \\
\hline 118.149 & 98.606 & 189.453 & 143.46 & 246.875 & 182.72 & 298.732 & 218.57 & 344.930 & 246.62 \\
\hline 120.979 & 100.03 & 191.652 & 144.68 & 248.787 & 184.45 & 300.568 & 219.03 & 346.536 & 247.95 \\
\hline 123.732 & 101.77 & 193.870 & 146.15 & 250.776 & 185.65 & 302.327 & 220.99 & 348.142 & 249.15 \\
\hline 126.486 & 103.70 & 196.086 & 147.16 & 252.688 & 187.45 & 304.086 & 222.70 & 349.672 & 249.61 \\
\hline 129.240 & 104.23 & 198.306 & 149.81 & 254.600 & 189.21 & 305.922 & 223.03 & 351.202 & 250.95 \\
\hline 131.840 & 106.50 & 200.456 & 150.85 & 256.512 & 190.12 & 307.681 & 224.30 & 352.732 & 250.98 \\
\hline 134.506 & 107.65 & 202.622 & 152.19 & 258.424 & 191.38 & 309.441 & 225.96 & & \\
\hline 137.114 & 109.84 & 204.808 & 153.88 & 260.260 & 192.48 & 311.200 & 226.77 & & \\
\hline 139.690 & 111.63 & 206.949 & 155.61 & 262.172 & 193.18 & 312.959 & 227.69 & & \\
\hline
\end{tabular}

表 3 氨茶碱的热容测量值

Table 3 Experimental molar heat capacity of aminophylline

\begin{tabular}{|c|c|c|c|c|c|c|c|c|c|}
\hline$T / \mathrm{K}$ & $\frac{C_{p, \mathrm{~m}}}{\left(\mathrm{~J} \cdot \mathrm{mol}^{-1} \cdot \mathrm{K}^{-1}\right)}$ & $T / \mathrm{K}$ & $\frac{C_{p, \mathrm{~m}}}{\left(\mathrm{~J} \cdot \mathrm{mol}^{-1} \cdot \mathrm{K}^{-1}\right)}$ & $T / \mathrm{K}$ & $\frac{C_{p, \mathrm{~m}}}{\left(\mathrm{~J} \cdot \mathrm{mol}^{-1} \cdot \mathrm{K}^{-1}\right)}$ & $T / \mathrm{K}$ & $\frac{C_{p, \mathrm{~m}}}{\left(\mathrm{~J} \cdot \mathrm{mol}^{-1} \cdot \mathrm{K}^{-1}\right)}$ & $T / \mathrm{K}$ & $\frac{C_{p, \mathrm{~m}}}{\left(\mathrm{~J} \cdot \mathrm{mol}^{-1} \cdot \mathrm{K}^{-1}\right)}$ \\
\hline 78.943 & 147.45 & 123.280 & 191.77 & 158.220 & 226.54 & 234.494 & 349.04 & 335.891 & 747.11 \\
\hline 81.615 & 149.83 & 125.150 & 192.72 & 159.870 & 228.73 & 240.039 & 363.52 & 340.282 & 775.58 \\
\hline 84.252 & 152.85 & 127.013 & 194.94 & 161.482 & 229.72 & 245.578 & 375.10 & 344.490 & 800.77 \\
\hline 86.809 & 155.39 & 128.895 & 196.10 & 163.101 & 231.48 & 251.082 & 390.23 & 348.479 & 821.95 \\
\hline 89.297 & 158.47 & 130.720 & 198.91 & 164.688 & 233.84 & 256.519 & 405.56 & 352.101 & 843.30 \\
\hline 91.676 & 160.77 & 132.527 & 200.27 & 166.269 & 235.92 & 261.921 & 421.77 & 355.468 & 864.48 \\
\hline 94.055 & 163.20 & 134.352 & 201.25 & 167.849 & 237.04 & 267.222 & 439.52 & 358.468 & 881.27 \\
\hline 96.399 & 165.78 & 136.125 & 203.56 & 169.454 & 239.69 & 272.420 & 455.78 & 360.261 & 897.15 \\
\hline 98.668 & 167.60 & 137.928 & 205.02 & 171.011 & 241.29 & 277.585 & 473.62 & 363.189 & 914.85 \\
\hline 100.862 & 169.36 & 139.659 & 206.60 & 172.592 & 242.93 & 282.716 & 490.87 & 364.469 & 925.81 \\
\hline 103.058 & 171.76 & 141.428 & 208.06 & 175.113 & 246.66 & 287.778 & 507.10 & 365.018 & 931.65 \\
\hline 105.221 & 173.66 & 143.159 & 210.17 & 180.138 & 252.68 & 292.807 & 528.34 & 365.091 & 933.11 \\
\hline 107.334 & 175.30 & 144.853 & 212.49 & 186.593 & 261.81 & 297.768 & 553.76 & 365.311 & 934.93 \\
\hline 109.434 & 178.59 & 146.547 & 213.73 & 192.916 & 270.19 & 302.729 & 573.93 & 366.262 & 941.69 \\
\hline 111.470 & 179.12 & 148.259 & 215.68 & 199.107 & 280.59 & 307.656 & 598.28 & 367.287 & 950.08 \\
\hline 113.501 & 181.64 & 149.953 & 217.82 & 205.242 & 290.70 & 312.514 & 618.21 & 368.202 & 954.45 \\
\hline 115.502 & 183.44 & 151.609 & 219.84 & 211.264 & 298.99 & 317.339 & 644.71 & 368.568 & 958.65 \\
\hline 117.472 & 185.96 & 153.303 & 221.71 & 217.211 & 309.83 & 322.096 & 669.53 & & \\
\hline 119.444 & 187.26 & 154.959 & 222.64 & 223.079 & 321.72 & 326.785 & 697.46 & & \\
\hline 121.380 & 189.28 & 156.577 & 224.99 & 228.826 & 335.47 & 331.406 & 723.95 & & \\
\hline
\end{tabular}




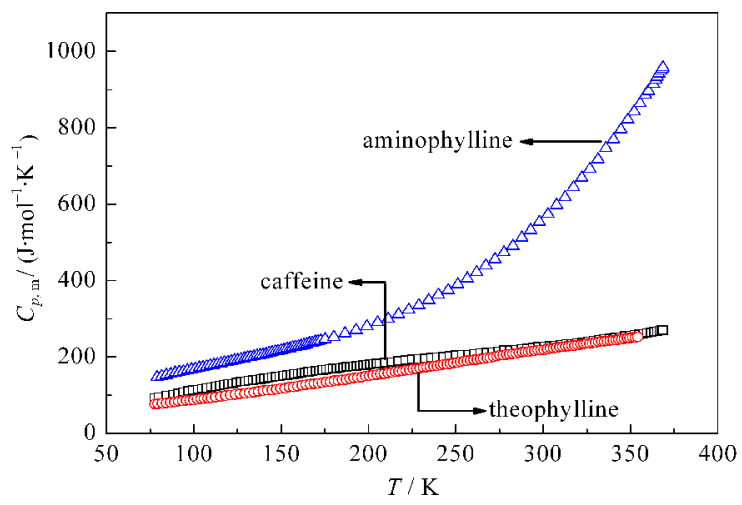

图 2 咖啡因、茶碱和氨茶碱的 $C_{p, \mathrm{~m}}$ 与温度的关系曲线

Fig.2 $C_{p, \mathrm{~m}}-T$ curves of caffeine, theophylline, and aminophylline

$C_{p, \mathrm{~m}}=51.50658+169.1427 t+2292.884 t^{2}-$ $3306.370 t^{3}$

而对于氨茶碱, 其摩尔热容与折合温度的关系式为:

$C_{p, \mathrm{~m}}=18.63441+2377.982 t-12172.41 t^{2}+$ $34292.11 t^{3}$

利用上述关系式(1)-(3)计算了这三种药物的摩 尔热容值, 结果表明其计算值与实验值的相对误差 均在 $\pm 0.8 \%$ 以内, 表明拟合得到的这三种药物的摩 尔热容与温度的关系式能很好地反映它们的真实热 容值.

计算得到这三种药物在 $298.15 \mathrm{~K}$ 时的热容分 别为 $226.49 \mathrm{~J} \cdot \mathrm{K}^{-1} \cdot \mathrm{mol}^{-1}$ (咖啡因) 、 $218.13 \mathrm{~J} \cdot \mathrm{K}^{-1} \cdot \mathrm{mol}^{-1}$ (茶碱)和 $554.78 \mathrm{~J} \cdot \mathrm{K}^{-1} \cdot \mathrm{mol}^{-1}$ (氨茶碱).

文献报导 ${ }^{[15]}$ 的 $\beta$ 型咖啡因 $25{ }^{\circ} \mathrm{C}(298.15 \mathrm{~K})$ 的摩 尔热容分别为 $232 \mathrm{~J} \cdot \mathrm{K}^{-1} \cdot \mathrm{mol}^{-1}$ (差热分析测量的外 推值)和 $228 \mathrm{~J} \cdot \mathrm{K}^{-1} \cdot \mathrm{mol}^{-1}$ (滴定量热测量的估算值); $100{ }^{\circ} \mathrm{C}(373.15 \mathrm{~K})$ 的摩尔热容为 $(271 \pm 9) \mathrm{J} \cdot \mathrm{K}^{-1} \cdot \mathrm{mol}^{-1}$. 不同方法测得的 $\alpha$ 型咖啡因在 $100{ }^{\circ} \mathrm{C}(373.15 \mathrm{~K})$ 的 摩尔热容值在 285 到 $343 \mathrm{~J} \cdot \mathrm{K}^{-1} \cdot \mathrm{mol}^{-1}$ 之间. 根据本 文得到的热容与折合温度的关系式(1), 计算得到咖 啡因在 $298.15 \mathrm{~K}$ 的摩尔热容为 $226.49 \mathrm{~J} \cdot \mathrm{K}^{-1} \cdot \mathrm{mol}^{-1}$; $373.15 \mathrm{~K}$ 的摩尔热容为 $272.92 \mathrm{~J} \cdot \mathrm{K}^{-1} \cdot \mathrm{mol}^{-1}$, 这与文 献报道的 $\beta$ 型咖啡因的热容值 ${ }^{[15]}$ 相吻合, 可见, 本文 测定的为 $\beta$ 型咖啡因的摩尔热容. 而茶碱和氨茶碱 的摩尔热容目前尚无文献报道.

依据方程(1)-(3), 可计算咖啡因、茶碱和氨茶碱 相对于 $298.15 \mathrm{~K}$ 的焓变值 $\left(H_{T}-H_{298.15 \mathrm{~K}}\right)$ 和熵变值 $\left(S_{T}-\right.$ $S_{298.15 \mathrm{~K}}$, , 其中:

$$
H_{T}-H_{298.15 \mathrm{~K}}=\int_{298.15 \mathrm{~K}}^{T} C_{p, \mathrm{~m}} \mathrm{~d} T
$$

$$
S_{T}-S_{28.15 \mathrm{~K}}=\int_{29.15 \mathrm{~K}}^{T} \frac{C_{p, \mathrm{~m}}}{T} \mathrm{~d} T
$$

\section{2 咖啡因、茶碱和氨茶碱的热分析}

采用热综合分析仪对咖啡因、茶碱和氨茶碱进 行热分析实验, 分别获得它们的热失重(TG)曲线和 差示扫描量热(DSC)曲线, 结果见图 3.

由图 3 可知, 咖啡因和茶碱仅有一个失重阶段. 咖啡因的失重温度范围为 512-590 K, 失重率为 $98.41 \%$, 因实验结束后在炉壁及吊杆上观察到了白 色针状晶体, 可推断该失重是因咖啡因蒸发而非分 解所致. 茶碱失重的温度范围为 550-636 K, 失重量
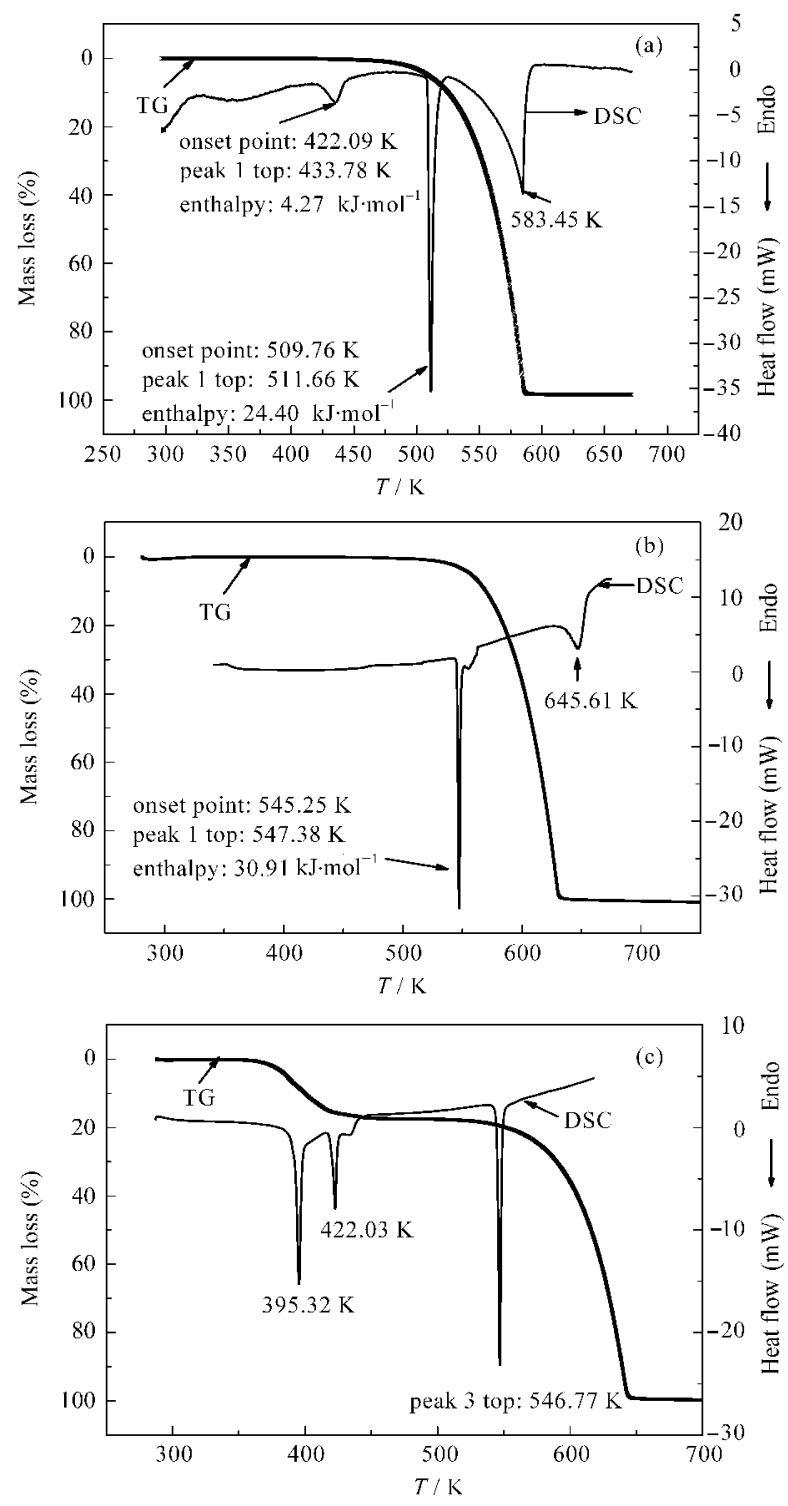

图 3 咖啡因(a)、茶碱(b)和氨茶碱(c)的 TG-DSC 曲线

Fig.3 TG-DSC curves of caffeine (a), theophylline (b), and aminophylline (c) 
表 4 咖啡因和茶碱相变过程的热力学参数

Table 4 Thermodynamics parameters of caffeine and theophylline in phase change processes

\begin{tabular}{|c|c|c|c|c|c|c|c|c|c|c|}
\hline \multirow{4}{*}{ Name } & \multicolumn{5}{|c|}{ Solid-solid phase change } & \multicolumn{5}{|c|}{ Fusion phase change } \\
\hline & \multirow{2}{*}{\multicolumn{2}{|c|}{$T_{\mathrm{m}} / \mathrm{K}$}} & \multirow{2}{*}{\multicolumn{2}{|c|}{$\frac{\Delta H_{\mathrm{m}}}{\left(\mathrm{kJ} \cdot \mathrm{mol}^{-1}\right)}$}} & \multirow{3}{*}{$\frac{\frac{\Delta S_{\mathrm{m}}}{\left(\mathrm{J} \cdot \mathrm{mol}^{-1} \cdot \mathrm{K}^{-1}\right)}}{\text { this work }}$} & \multirow{2}{*}{\multicolumn{2}{|c|}{$T_{\mathrm{m}} / \mathrm{K}$}} & \multirow{2}{*}{\multicolumn{2}{|c|}{$\frac{\Delta H_{\mathrm{m}}}{\left(\mathrm{kJ} \cdot \mathrm{mol}^{-1}\right)}$}} & \multirow{3}{*}{$\frac{\Delta S_{\mathrm{m}}}{\frac{\left(\mathrm{J} \cdot \mathrm{mol}^{-1} \cdot \mathrm{K}^{-1}\right)}{\text { this work }}}$} \\
\hline & & & & & & & & & & \\
\hline & this work & Ref. & this work & Ref. & & this work & Ref. & this work & Ref. & \\
\hline \multirow[t]{2}{*}{ caffeine } & $422.09 \pm 0.84$ & $426^{[14]}$ & $4.27 \pm 0.06$ & $3.93^{[14]}$ & $10.11 \pm 0.14$ & $509.76 \pm 0.07$ & $512^{[14]}$ & $24.40 \pm 0.31$ & $23.41^{[14]}$ & $47.86 \pm 0.61$ \\
\hline & & $414.15^{[16]}$ & & $4.1^{[16]}$ & & & $509.25^{[16]}$ & & $21.6^{[16]}$ & \\
\hline theophylline & - & - & - & - & - & $545.25 \pm 0.11$ & $544.35^{[16]}$ & $30.91 \pm 0.95$ & $30.9^{[16]}$ & $56.69 \pm 1.74$ \\
\hline
\end{tabular}

为 $99.54 \%$, 实验结束后仅在坩埚上有少量的积碳, 炉壁及吊杆上未见任何残留物, 可推断茶碱的失重 是因其分解所致. 氨茶碱有二个失重阶段, 第一个失 重阶段的温度范围为 370-471 K, 其失重量为 $16.9 \%$, 根据其失重量可推断在此过程中脱去一分 子水和一分子乙二胺(按此历程热解的理论失重率约 为 $17.8 \%$ ), 第二个失重阶段的温度范围为 560-652 $\mathrm{K}$, 失重量为 $82.58 \%$. 由此可知, 它们的热稳定顺序 为氨茶碱 $<$ 咖啡因 $<$ 茶碱.

观察咖啡因的 DSC 曲线形状, 该曲线有三个吸 热峰. 其中第一个和第二个吸热峰均出现在失重之 前, 表明这两个吸热峰均为相变所引起的. 根据文 献 ${ }^{[14]}$, 咖啡因的固-固相转变温度 $426 \mathrm{~K}$ 、熔点 $512 \mathrm{~K}$, 可知第一个峰为咖啡因的晶型转变所引起的; 第二 个峰为固态咖啡因转变为液态咖啡因的相变峰; 第 三个吸热峰出现在失重阶段. 由此可知, 咖啡因受热 变化过程为: 咖啡因晶体受热后, 约在 $422 \mathrm{~K}$ 发生 固-固相变; 约在 $509 \mathrm{~K}$ 咖啡因由固态转变为液态, $512 \mathrm{~K}$ 以后开始蒸发.

茶碱的 DSC 曲线存在两个吸热峰. 第一个吸热 峰出现在它失重之前, 依据文献 ${ }^{[16]}$ 可知, 茶碱的熔点 为 $544.7 \mathrm{~K}$, 因此可以推测这个峰为它的熔化峰; 而 第二个吸热峰出现在失重阶段, 则此峰是由其热失 重引起的. 因此茶碱受热变化过程为: 当升温到 545 $\mathrm{K}$ 左右时, 茶碱由固态转变液态; 继续升温到 $550 \mathrm{~K}$ 左右, 液态茶碱开始分解.

氨茶碱的 DSC 曲线有三个吸热峰, 第一个峰的 峰温约为 $395 \mathrm{~K}$, 第二个峰的峰温约为 $422 \mathrm{~K}$, 这两 个吸热峰均出现在它的第一个失重阶段; 第三个峰 出现在其 TG 曲线的第二个平台内, 与茶碱熔化峰 的峰温一致. 因此氨茶碱受热变化过程为: 当温度 升到 $370 \mathrm{~K}$ 时, 氨茶碱脱去一分子水, 紧接着脱去一 分子乙二胺, 此时氨茶碱变为固态茶碱; 温度继续 升到约 $547 \mathrm{~K}$ 时, 茶碱由固态转变液态; 继续升温, 茶碱将分解.
根据 DSC 曲线可准确获得咖啡因和茶碱的相 变温度 $\left(T_{\mathrm{m}}\right)$ 、相变焓 $\left(\Delta H_{\mathrm{m}}\right)$ 和相变熵 $\left(\Delta S_{\mathrm{m}}\right)$ (见表 4$)$. 结 果表明: 本文测定的数值与文献报道的数值 ${ }^{[14,16]}$ 基本 一致.

综合 TG 和 DSC 分析结果, 可知咖啡因的起始 热失重温度和熔点均低于茶碱. 这是因为咖啡因 $\mathrm{N}$ 原子上的氢被甲基取代造成的. 就氨茶碱而言, 热分 析结果表明其脱水和脱乙二胺基本上是同时发生. 因此, 氨茶碱在保存过程中, 一旦脱水, 就将失去其 特有的药理作用.

\section{3 理论计算}

上述实验结果显示: 咖啡因和茶碱由于与 $\mathrm{N}$ 原 子相连的基团不同, 导致其热化学性质不同. 通过 Hirshfeld 电荷分析可知, 与 $\mathrm{H}$ 连接的 $\mathrm{N}$ 原子的电荷 数为 $0.114 e$, 而与一 $\mathrm{CH}_{3}$ 连接的 $\mathrm{N}$ 原子的电荷数仅 为 $0.024 e$. 此结果表明: 与一 $\mathrm{CH}_{3}$ 相比, $\mathrm{N}$ 原子能从 $\mathrm{H}$ 原子处获得更多的电子, 即 $\mathrm{N}$ 和 $\mathrm{H}$ 的作用强于 $\mathrm{N}$ 和一 $\mathrm{CH}_{3}$, 揭示了茶碱分子更稳定. 而茶碱以及咖啡 因分子间的相互作用计算结果显示, 它们均可形成 分子间氢键. 咖啡因分子间的氢键 $(\mathrm{O} \cdots \mathrm{H}-\mathrm{C})$ 键长 为 $0.220 \mathrm{~nm}$ 、键能为 $5.3 \mathrm{~kJ} \cdot \mathrm{mol}^{-1}$, 茶碱分子间的氢 键 $(\mathrm{O} \cdots \mathrm{H}-\mathrm{N})$ 键长为 $0.171 \mathrm{~nm}$ 、键能为 $19.7 \mathrm{~kJ} \cdot \mathrm{mol}^{-1}$, 这表明茶碱分子间的作用要强于咖啡因. 上述计算 结果显示由于- $\mathrm{CH}_{3}$ 的取代使咖啡因分子内及分子 间的稳定性降低, 使得其热稳定性和熔点均低于茶 碱.

另外, 本文基于密度泛函理论的第一性原理计 算方法计算了咖啡因和茶碱的热容, 结果表明咖啡 因的热容值略大于茶碱. 以 $298.15 \mathrm{~K}$ 为例, 咖啡因 和茶碱的热容计算值分别为 210.31 和 $170.75 \mathrm{~J} \cdot \mathrm{K}^{-1}$. $\mathrm{mol}^{-1}$, 与实验结果的趋势相吻合.

\section{3 结 论}

对咖啡因、茶碱和氨茶碱进行了系统的热分析 和量热学研究, 采用低温绝热量热仪测量了这三种 
药物的热容值. 结果表明: 因氨茶碱是复合盐, 因此 其摩尔热容值最大, 而咖啡因的摩尔热容值略大于 茶碱. 本文利用最小二乘法对这三种药物的实验摩 尔热容值和折合温度进行拟合, 分别得到它们的摩 尔热容值与折合温度的关系式, 并依次获得了其有 关的热力学参数. 热分析结果表明: 这三种药物的 热稳定性顺序为氨茶碱<咖啡因<茶碱. 通过 DSC 测量, 获得了咖啡因和茶碱的相转变温度、相转变焓 和相转变熵, 这些数据与文献报道的结果相符. 咖啡 因和茶碱仅因 $\mathrm{N}$ 上的基团不同, 导致其热化学性质 不同. 本文通过基于密度泛函理论的第一性原理对 咖啡因和茶碱分子的结构稳定性进行了计算, 结果 表明由于甲基的取代, 造成咖啡因分子内及分子间 的稳定性降低, 引起其热化学性质上的改变.

\section{References}

1 Kassim, Z.; Greenough, A.; Rafferty, G. F. Eur. J. Pediatr., 2009, 168: 1491

2 Skouroliakou, M.; Bacopoulou, F.; Markantonis, S. L. J. Paediatr. Child Health, 2009, 45: 587

3 Zhu, M.; Sun, Y.; Guo, D. H.; Shi, L. China Pharmacy, 2007, 18: 1838 [朱 曼, 孙 艳, 郭代红, 石 莉. 中国药房, 2007, 18: 1838]

4 Farber, H. J. Curr. Opin. Pulm. Med., 2010, 16: 25

5 Raj, N. S.; Guleria, R.; Misra, A. J. Clin. Pharmacol., 2006, 46: 1070

6 Zhang, H.; Zhao, A. H.; Chen, N. J. Pharmaceutical and Clinical Research, 2009, 17: 160 [张 辉, 赵爱华, 陈乃江. 药学与临床 研究, 2009, 17: 160]

7 Li, Q.; Zhang, T. T.; Lv, W. Y. Eur. J. Med. Chem., 2009, 44: 1452

8 Mazurek, S.; Szostak, R. J. Pharm. Biomed. Anal., 2006, 40: 1235

9 He, X. J.; Liu, X. H.; Jia, S. S. Drug Standards of China, 2006, 7: 38 [赫修洁, 刘晓华, 贾首时. 中国药品标准, 2006, 7: 38]
10 Kunadharaju, S.; Savva, M. J. Chem. Eng. Data, 2010, 55: 103

11 Zhang, J.; Chen, D. H.; Yuan, Y. H.; Yang, T. M.; Qu, H. A. Acta Pharmaceutica Sinica, 2000, 35: 44］张＼cjkstart健, 陈栋华, 袁誉洪, 杨天鸣, 睢鸿岸. 药学学报, 2000, 35: 44]

12 Neto, H. S.; Novak, C.; Matos, J. R. J. Therm. Anal. Cal., 2009, 97: 367

13 Dang, Z.; Song, L. X.; Pan, S. Z.; Wang, M. Acta Phys. -Chim. Sin., 2009, 25: 1059 [党 政, 宋乐新, 潘淑臻, 王 莽. 物理化学学 报, 2009, 25: 1059]

14 Cesàro, A.; Starec, G. J. Phys. Chem., 1980, 84: 1345

15 Bothe, H.; Cammenga, H. K. J. Therm. Anal., 1979, 16: 267

16 Bruns, S.; Reichelt, J.; Cammenga, H. K. Thermochim. Acta, 1984, 72: 31

17 Dong, J. X.; Li, Q.; Tan, Z. C.; Zhang, Z. H.; Liu, Y. J. Chem. Thermodyn., 2007, 39: 108

18 Yoshihashi, Y.; Makita, M.; Yamamura, S.; Fukuoka, E.; Terada, K. Chem. Pharm. Bull., 1998, 46: 1148

19 Sarma, P. D.; Nandita, G. D.; Terrance, P. K.; Theodore, D. S. Int. J. Pharm., 1995, 114: 247

20 Druzhinina, A. I.; Varushchenko, R. M.; Troyanov, S. I.; Sidorov, L. N. J. Chem. Thermodyn., 2010, 42: 165

21 Liu, P.; Xiong, W.; Hu, S. Z.; Li, X.; Tan, Z. C. Acta Phys. -Chim. Sin., 2009, 25: 2417 [刘 鹏, 熊 伟, 胡善洲, 李 䂀, 谭志诚. 物理化学学报, 2009, 25: 2417]

22 Paukov, I. E.; Kovalevskaya, Y. A.; Boldyreva, E. V.; Drebushchak, V. A. J. Therm. Anal. Cal., 2009, 98: 873

23 Xu, F.; Sun, L. X.; Tan, Z. C.; Liang, J. G.; Zhang, T. J. Therm. Anal. Cal., 2006, 83: 187

24 Chirico, R. D.; Steele, W. V. J. Chem. Thermodyn., 2009, 41: 392

25 Uchida, A.; Moriya, Y.; Kawaji, H.; Atake, T.; Fukuhara, M.; Kimura, H.; Inoue, A. J. Chem. Eng. Data, 2009, 54: 2033

26 Archer, D. G. J. Phys. Chem. Ref. Data, 1993, 22: 1441

27 (a) Hohenberg, P.; Kohn, W. Phys. Rev. B, 1964, 136: 864 (b) Kohn, W.; Sham, L. J. Phys. Rev. A, 1965, 140: 1133

28 Delley, B. J. Chem. Phys., 2000, 113: 7756

29 Perdew, J. P.; Wang, Y. Phys. Rev. B, 1992, 45: 13244 